\title{
Efeitos de diferentes fontes de energia sobre taxa ovulatória, fertilidade e sobrevivência embrionária em marrãs cíclicas
}

[Effects of different energy sources on ovulation rate, pregnancy rate and embryo survival in cyclic gilts]

\author{
G.S. Machado ${ }^{1}$, D.O. Fontes ${ }^{2}$, F.R.C.L. Almeida ${ }^{3}$, A.L.C.C. Borges ${ }^{2}$, \\ M.A. Silva ${ }^{2}$, V.F. Oliveira ${ }^{4}$, T.C.T. Silva ${ }^{4}$ \\ ${ }^{1}$ Aluno de pós-graduação - EV-UFMG - Belo Horizonte, MG \\ ${ }^{2}$ Escola de Veterinária - UFMG - Belo Horizonte, MG \\ ${ }^{3}$ Iinstituto de Ciências Biológicas - UFMG - Belo Horizonte, MG \\ ${ }^{4}$ Médico veterinário autônomo
}

\begin{abstract}
RESUMO
Cinqüenta e quatro marrãs cíclicas, uniformizadas quanto à linhagem, família, ganho de peso, espessura de toucinho, peso, precocidade sexual, número de cios e escore clínico, foram alocadas em dois grupos experimentais com dietas isocalóricas, isoprotéicas e isolisínicas. Duas fontes de energia foram testadas: amido de milho (T1) e óleo de soja (T2). Sincronizou-se o segundo estro com allyl-trenbolone, para inseminação no terceiro estro. Foi realizada cateterização não cirúrgica em 21 marrãs, submetidas a dois ciclos de coleta para dosagem de glicose e insulina, aos 14 e 21 dias do ciclo. Todas as marrãs foram abatidas aos 28,6 dias de gestação média, para análises biométricas do trato reprodutivo. Marrãs do T1 apresentaram maior taxa ovulatória em relação às do T2 (16,52 vs 14,70, P<0,01). Não houve diferença entre os tratamentos nas taxas de prenhez e sobrevivência embrionária. É possível alterar a eficiência reprodutiva por intermédio de manipulação dietética, mesmo em marrãs em estado anabólico. O uso do amido de milho na fase pré-cobertua melhorou a eficiência reprodutiva dos animais avaliados.
\end{abstract}

Palavras-chave: marrãs, insulina, energia, flushing

\begin{abstract}
Fifty four cyclic gilts were randomly selected and uniformized according to genetic background, litter of origin, weight gain, backfat, number of cycles and clinical score. Gilts were alloted to one of two groups fed isocaloric, isoproteic and isolysinic diets. Two energy sources were tested: corn starch (T1) and soybean oil (T2). Second estrus was synchronized with oral allyl-trenbolone, so that insemination was carried out at third estrus. Indweeling catheters were implanted by non-surgical technic in 21 gilts, which were submitted to consecutive blood samplings for glicose and insulin determination. Timing of ovulation was estimated by means of ultrasonography. All gilts were slaughtered at an average gestation lenght of 28.6 days. Starch-fed gilts (T1) showed higher ovulation rates than T2 gilts (16.52 vs 14.70; P<0.01). There was no effect of treatments on pregnancy rate and embryo survival. Results indicate it is possible to manipulate reproductive efficiency through diet even in anabolic experimental models like cyclic gilts. Feeding starch as main energy source during pre-mating flushing phase improved reproductive efficiency of cyclic gilts.
\end{abstract}

Keywords: gilts, insulin, energy, flushing

Recebido em 23 de agosto de 2007

Aceito em 5 de maio de 2008

Endereço para correspondência (corresponding address)

Rua Jornalista Jair Silva, 467/102 - 30310-290 - Belo Horizonte, MG,

E-mail: glauber.machado@terra.com.br 


\section{INTRODUÇÃO}

$\mathrm{Na}$ ampliação de plantéis ou mesmo na implantação de novas unidades de produção, a marrã representa a unidade fundamental dos sistemas produtivos. $\mathrm{O}$ bom desempenho produtivo das marrãs interfere não somente no resultado imediato dos plantéis recémestabelecidos, mas influi também na sua produtividade futura.

A nutrição específica de marrãs no período que antecede sua primeira cobrição ou inseminação já é prática consolidada em boa parte da indústria suinícola, uma vez que diversos autores já demonstraram que altos níveis de energia nessa fase estão relacionados à melhoria do desempenho reprodutivo (Rhodes, 1991; Murgas, 1994; Ashworth et al., 1999; Ferguson et al., 2003; Brustolini et al., 2004). Essa prática é mundialmente conhecida como flushing nutricional. Todavia, a compreensão dos efeitos de diferentes fontes de energia dietética nessa fase ainda carece de maior conhecimento científico.

Considerando o papel relevante que a insulina pode desempenhar nas interações entre a nutrição e a reprodução de marrãs, pode-se inferir que dietas que efetivamente promovam o aumento no nível de insulina plasmática venham a representar uma importante ferramenta para a melhoria da eficiência reprodutiva dos rebanhos. O princípio básico para a definição dos tratamentos experimentais está fundamentado na hipótese de que uma das fontes energéticas (carboidratos) promoveria maior resposta insulínica aguda, quando comparada à outra fonte testada (lípides), segundo van den Brand (2000).

Assim, o objetivo do trabalho foi avaliar o desempenho reprodutivo de marrãs cíclicas ao se utilizarem dietas isoenergéticas baseadas em duas diferentes fontes de energia.

\section{MATERIAL E MÉTODOS}

Após a primeira triagem e seleção, 64 marrãs foram transferidas das instalações de recria e alojadas nas instalações de reposição de plantel. Estas foram posteriormente alojadas em gaiolas metálicas individuais com $2,20 \mathrm{~m}$ de comprimento por $0,62 \mathrm{~m}$ de largura, divididas em grupos de irmãs completas, oriundas da mesma leitegada (pai e mãe idênticos) e distribuídas em delineamento experimental de blocos inteiramente ao acaso, em que cada bloco representou uma dupla de irmãs de mesma leitegada de origem, destinadas a cada um dos dois tratamentos. O protocolo experimental incluiu a sincronização do estro de todas as leitoas para que essa variável fosse eliminada, por intermédio da administração oral do progestágeno sintético ${ }^{1}$, iniciada entre 12 e 14 dias após a manifestação do primeiro cio, na dose de $20 \mathrm{mg} / \mathrm{dia}$, de forma individual e pela via oral, misturado a uma pequena quantidade de ração. O segundo estro foi alcançado entre cinco e seis dias após a suspensão do uso do progestágeno sintético, em $96 \%$ das marrãs, incluídas nos respectivos tratamentos. No oitavo dia subseqüente ao dia do segundo estro de cada leitoa, iniciou-se o fornecimento das dietas experimentais: tratamento 1 (grupo flushing-carboidratos) composto por marrãs alimentadas à vontade, entre o dia oito do terceiro ciclo estral e o cio subseqüente, com dieta cuja fonte adicional de energia foi predominantemente constituída por carboidratos (20\% amido de milho); tratamento 2 (grupo flushing-lípides), formado por marrãs alimentadas à vontade, entre o dia oito do terceiro ciclo estral e o cio subseqüente, com dieta cuja fonte adicional de energia foi predominantemente lipídica (óleo refinado de soja, em quantidade exata para substituir a energia fornecida pela fonte de amido incluída no tratamento 1, completando-se a diferença quantitativa com ingrediente inerte).

As dietas isocalóricas, isoproteícas e isolisínicas eram constituídas pela mesma matéria-prima básica (milho, farelo de soja e farelo de trigo). O consumo foi monitorado para tornar a ingestão diária de energia metabolizável similar, para ambos os tratamentos. $\mathrm{O}$ arraçoamento foi realizado duas vezes ao dia, às $9 \mathrm{~h}$ e $30 \mathrm{~min}$ e às $16 \mathrm{~h}$ e $30 \mathrm{~min}$. A diferença essencial entre os tratamentos foi a utilização do amido de milho ou do óleo vegetal (óleo de soja), como fonte energética adicional à matéria-prima tradicional, em proporções idênticas para ambas as rações. A composição das rações encontra-se na Tab. 1.

Os níveis nutricionais utilizados nas rações experimentais foram condizentes com o conceito de alta energia para o período de flushing em marrãs cíclicas, (Tab. 2).

${ }^{1}$ Regumate ${ }^{\circledR}$ Hoechst-Rousell Vet., Alemanha 
Tabela 1. Composição básica das rações experimentais, expressa em porcentagem da matéria natural, segundo os tratamentos

\begin{tabular}{lcc}
\multicolumn{1}{c}{ Ingrediente } & Tratamento 1 (\%) & Tratamento 2 (\%) \\
\hline Milho, grão moído & 45,496 & 45,496 \\
Farelo de soja & 27,074 & 27,074 \\
Farelo de trigo & 4,000 & 4,000 \\
Calcário calćítico & 0,937 & 0,937 \\
Fosfato bicálcico & 1,625 & 1,625 \\
Sal (NaCl) & 0,369 & 0,369 \\
Suplem. vitaminas e aminoácidos & 0,400 & 0,400 \\
Suplemento mineral & 0,100 & 0,100 \\
Amido de milho & 20,000 & - \\
Óleo de soja refinado & - & 8,611 \\
Caulim (inerte) & - & 11,389 \\
\hline Total & 100 & 100 \\
\hline
\end{tabular}

Tabela 2. Níveis nutricionais das rações experimentais, expressos em porcentagem da matéria natural, segundo os tratamentos

\begin{tabular}{lcc}
\multicolumn{1}{c}{ Componente da análise } & Tratamento 1 (\%) & Tratamento 2 (\%) \\
\hline Proteína bruta & 17,0 & 17,0 \\
Fibra bruta & 2,85 & 2,85 \\
Lisina & 0,892 & 0,892 \\
Metionina + Cistina & 0,533 & 0,533 \\
Treonina & 0,652 & 0,652 \\
Triptofano & 0,211 & 0,211 \\
Cálcio & 0,84 & 0,84 \\
Fósforo total & 0,607 & 0,607 \\
Fósforo disponível & 0,40 & 0,40 \\
Sódio & 0,180 & 0,180 \\
Energia metabolizável $(\mathrm{kcal} / \mathrm{kg})$ & 3162 & 3162 \\
Amido & 51,47 & 32,25 \\
Extrato etéreo & 2,119 & 9,88 \\
\hline
\end{tabular}

Durante todo o período de alojamento individual, as marrãs foram diariamente expostas ao macho para detecção de sinais exteriores de cio, duas vezes ao dia, sempre nos mesmos horários. O protocolo de sincronização de cio adotado obteve êxito em 54 das 55 marrãs disponíveis. As marrãs foram inseminadas com doses de sêmen originadas de um mesmo grupo de cachaços adultos, submetidos à avaliação andrológica anterior ao experimento. Todas as doses seminais utilizadas foram padronizadas para uma concentração única de três bilhões de células viáveis, mediante contagem em Câmara de Neubauer. Os parâmetros mínimos foram: $80 \%$ de motilidade progressiva e incidência de defeitos totais (maiores e menores) nunca acima de $10 \%$ das células totais do ejaculado. O diluente utilizado foi o Beltsville Thawing Solution (BTS). Uma vez inseminadas, as leitoas passaram imediatamente ao plano nutricional de gestação, recebendo dois quilogramas diários de uma dieta típica para essa fase da produção. Os tratamentos foram encerrados no momento da primeira inseminação artificial. Deste momento até o dia do abate experimental, todas as leitoas foram submetidas a um plano nutricional único.

$\mathrm{O}$ abate das marrãs foi programado para ocorrer entre 28 e 30 dias de gestação. A partir de 15 dias após a inseminação, foi restabelecido o manejo de detecção diária de cio, duas vezes ao dia, com o objetivo de identificar as leitoas que retornassem ao estro. Os animais não fertilizados foram excluídos da análise, visto que os dados ovarianos avaliados já não mais seriam representativos dos tratamentos nutricionais aplicados. Durante o abate experimental, foi coletado todo o trato reprodutivo que inclui 
vagina, útero gravídico e ovários para mensuração de todos os dados relativos ao desempenho reprodutivo de cada um dos animais abatidos. A análise final dos dados contemplou 42 unidades experimentais, sendo 21 blocos e dois tratamentos por bloco.

O delineamento experimental foi em blocos ao acaso, sendo que cada grupo de irmãs completas constituiu um bloco, com dois tratamentos por bloco. Cada leitoa representou uma unidade experimental, sendo que, para cada marrã alocada a um dos tratamentos, uma leitoa-irmã foi alocada ao outro tratamento testado. Os dados obtidos foram submetidos à análise de variância por meio do programa SAEG (Sistema... 2004).

\section{RESULTADOS E DISCUSSÃO}

Na Tab. 3 apresentam-se os dados de peso vivo, espessura de toucinho, consumo médio diário e tempo médio de gestação, ao final do tratamento, segundo as dietas experimentais.

Tabela 3. Peso vivo final (PV), espessura de toucinho final (ET), consumo médio diário de ração (CMD) e tempo de gestação (TG) no momento do abate de marrãs cíclicas, submetidas aos tratamentos com amido de milho (T1) e com óleo vegetal (T2)

\begin{tabular}{ccc}
\hline Média & T1 & T2 \\
\hline PV final $(\mathrm{kg})$ & $137,99 \pm 3,96$ & $136,97 \pm 9,35$ \\
ET final $(\mathrm{mm})$ & $28,76 \pm 3,57$ & $28,62 \pm 4,87$ \\
CMD (g/dia) & $3048,52 \pm 492,4$ & $3007,81 \pm 445,5$ \\
TG (dias) & $28,62 \pm 0,5$ & $28,62 \pm 0,8$ \\
\hline
\end{tabular}

${ }^{\mathrm{O}} \mathrm{O}$ tempo de gestação não é resultante de ação dos tratamentos, portanto não há análise estatística dessa variável

A análise dos dados da Tab. 3 remete ao procedimento de seleção dos animais experimentais. Os animais alocados no experimento derivam de um grupo maior de marrãs, o qual foi submetido a sucessivas avaliações fenotípicas para seleção do grupo final,, com base na uniformidade de seu desempenho durante as fases pré e póspuberdade. Não houve efeito dos tratamentos $(\mathrm{P}>0,05)$ sobre o peso final, nem tampouco sobre a espessura de toucinho ao final dos tratamentos. Este resultado era esperado, de fato, pois, ao contrário de outros autores (Beltranena et al., 1993; Almeida et al., 2001; Ferguson et al., 2003), não foi induzida neste experimento qualquer alteração no padrão de consumo das marrãs. Ao contrário, o consumo dos animais foi semelhante $(\mathrm{P}>0,05)$ entre os dois tratamentos, em que prevaleceu o fornecimento à vontade das dietas experimentais isoenergéticas. Segundo Azain (2000), dietas com alta participação de gordura, mesmo que isocalóricas em relação a dietas mais ricas em carboidratos, implicarão maior energia líquida disponível aos animais que as consomem, pelo menor incremento calórico e pela redução na taxa de passagem da digesta. Assim, a dieta contendo $8,6 \%$ de óleo de soja poderia, a princípio, propiciar um estado ainda mais anabólico nos animais do T2, em relação aos do T1. Esse efeito "extra-calórico" da dieta rica em lipídeos não resultou em maior ganho de peso ou em maior deposição de gordura. Isso ocorreu, provavelmente porque essa diferença em potencial de energia líquida das dietas não foi suficiente para promover tais alterações, em níveis minimamente detectáveis pelos métodos empíricos utilizados. Pode-se associar à interpretação outros fatores hipotéticos. Em primeiro lugar, o período relativamente curto dos tratamentos (14 dias) pode ter interagido com a pouca diferença de energia líquida potencial, e ambos teriam, assim, limitado as possibilidades de ação do eixo somatotrópico sobre síntese tecidual e deposição de gordura. E, por último, pode-se ainda supor que as marrãs utilizadas neste modelo experimental estiveram, durante todo o tempo, em estado absortivo e fortemente anabólico. Portanto, há excedente de combustíveis oxidáveis (glicose e ácidos graxos) e seu direcionamento para glicogenogênese e lipogênese, principalmente no fígado e nos adipócitos, respectivamente. Entretanto, conforme já demonstrado em porcas alimentadas à vontade durante a gestação (Weldon et al., 1994), períodos muito prolongados de situação anabólica intensa poderiam induzir a ocorrência de resistência insulínica nos tecidos, provavelmente por redução do número ou da afinidade de receptores insulínicos em alvos periféricos (adipócito, por exemplo). Essa 
resistência insulínica, também entendida como intolerância à glicose, compõe a fisiopatologia da diabete gestacional, amplamente evidenciada em mulheres e também em suínos (Weldon et al., 1994). Em síntese, o desenvolvimento de resistência insulínica significa a necessidade de maior concentração de insulina para promover o mesmo efeito metabólico, antes alcançado com concentrações insulínicas mais baixas. Todavia, a possibilidade de ocorrência desse mecanismo nas leitoas utilizadas neste experimento é apenas hipotética e, até onde se sabe, ainda não foi validada pela literatura científica. A metodologia adotada neste experimento não permite conclusões definitivas a esse respeito.

A comparação de dados de ganho de peso ou espessura de toucinho em modelos experimentais de curto prazo, na fase pré-cobertura, deve ser realizada de forma crítica. Isto porque, na maioria dos trabalhos revisados, foi aplicado algum nível de restrição alimentar quantitativa ou qualitativa. Flowers et al. (1989) alimentaram um grupo-controle com 5400kcalEM/dia, enquanto o grupo flushing recebeu $11.000 \mathrm{kcalEM} / \mathrm{dia}$. Os autores relataram diferença significativa no ganho de peso entre os tratamentos, mesmo com um período experimental de apenas 14 dias, exatamente a mesma duração dos tratamentos testados neste trabalho. Embora os tratamentos não tenham influenciado a espessura de toucinho final, houve efeito significativo da leitegada de origem sobre essa característica corporal $(\mathrm{P}=0,017)$, indicando a participação significativa de um componente genético na determinação dessa variável de alta herdabilidade. Quanto aos dados aparentemente elevados de espessura de toucinho ao final do experimento, cabe considerar que os animais experimentais eram originários da linhagem materna de um programa genético, portanto submetidos a processo de seleção direcionado para características reprodutivas, em detrimento da deposição de tecido magro na carcaça. Além disso, as marrãs foram alimentadas à vontade durante toda a fase de crescimento, com ração úmida, o que pode resultar em aumento na deposição de gordura dorsal (Whittemore, 1998).

A Tab. 4 representa um estudo de dispersão de freqüência para a ocorrência de prenhez entre os tratamentos. Não houve efeito dos tratamentos sobre a taxa de concepção (ou taxa de prenhez) após a inseminação artificial. Do mesmo modo,
Brustolini et al. (2004) descreveram taxas de fertilização equivalentes em marrãs submetidas a diferentes níveis energéticos e fontes lipídicas, a partir dos $60 \mathrm{~kg}$ de peso vivo aproximados. Considerando que, em espécies multíparas, o desafio metabólico mais significativo está na quantidade e na qualidade dos embriões concebidos, e não na simples prenhez ou concepção em si, a situação fortemente anabólica das marrãs deste experimento não justificaria diferenças nas taxas de concepção. A diferença numérica encontrada entre os tratamentos, quanto à taxa de prenhez $(92,00 \% \quad x \quad 84,62 \% ; \quad \mathrm{P}>0,05), \quad$ foi considerada como casualidade experimental.

Tabela 4. Taxa de prenhez, expressa em porcentagem, em marrãs cíclicas submetidas aos tratamentos com amido de milho (T1) e com óleo vegetal (T2)

\begin{tabular}{ccc}
\hline Tratamento & Positiva & Negativa \\
\hline T1 & 92,00 & 8,00 \\
T2 & 84,62 & 15,38 \\
\hline
\end{tabular}

Qui-quadrado $=0,6695(\mathrm{P}>0,05)$

A Tab. 5 contém os resultados obtidos para diversos parâmetros indicativos do desempenho reprodutivo, após o abate de todas as unidades experimentais, aos 28,6 dias de tempo médio de gestação. Os resultados ilustram significativa relação entre o tratamento dietético e as diversas respostas reprodutivas mensuradas. Em particular, a atividade ovariana parece ter sido fortemente influenciada pelos tratamentos, sendo que fêmeas do T1 apresentaram maior desempenho reprodutivo, quando comparado ao das fêmeas do T2. Estes resultados estão de acordo com os dados de Rodriguez-Marquez (1990) e Kemp et al. (1995), os quais encontraram efeitos positivos do aumento na participação de carboidratos na dieta sobre a função reprodutiva da fêmea suína. Os modelos experimentais adotados por autor, todavia, são muito variáveis e demandam análise mais detalhada das respostas obtidas e dos delineamentos aplicados a cada experimento. Os resultados observados por van den Brand et al. (2001) não indicaram efeito significativo das fontes de energia testadas (amido de milho + dextrose, comparados ao sebo) sobre as características uterinas, placentárias e embrionárias de porcas primíparas desmamadas. Mais uma vez, atenção deve ser dada na comparação de dados entre diferentes ensaios, pois as circunstâncias metabólicas que interferem nas respostas avaliadas são muito intensas, além da variabilidade intrínseca às respostas reprodutivas, em geral. 
Tabela 5. Número total de corpos lúteos (NTCL), peso total dos dois ovários (PTO), número total de embriões (NTE) e taxa de sobrevivência embrionária (TSE), em marrãs cíclicas submetidas aos tratamentos com amido de milho (T1) e com óleo vegetal (T2), com seus respectivos coeficientes de variação $(\mathrm{CV})$ e probabilidade do erro $(\mathrm{P})$

\begin{tabular}{lcccc}
\multicolumn{1}{c}{ Média } & T1 & T2 & CV & P \\
\hline NTCL & $16,52 \mathrm{a}$ & $14,70 \mathrm{~b}$ & 12,75 & $<0,01$ \\
NTE & $13,95 \mathrm{a}$ & $12,32 \mathrm{~b}$ & 11,41 & $<0,01$ \\
TSE (\%) & $84,80 \mathrm{a}$ & $84,23 \mathrm{a}$ & $*$ & NS \\
\hline
\end{tabular}

Letras minúsculas comparam médias em uma mesma linha.

TSE = (número de embriões viáveis/número total de corpos lúteos) x 100

$\mathrm{NS}=$ Não significativo $(\mathrm{P}>0,07)$

* Dado não disponível

Não houve efeito significativo dos tratamentos sobre a taxa de sobrevivência embrionária. A análise da sobrevivência embrionária merece algumas considerações, mesmo tendo-se constatado que a fonte de energia utilizada na dieta flushing não influenciou a mortalidade precoce dos embriões. Por tratar-se de variável influenciada pela qualidade intrínseca do embrião, pela condição metabólica e endócrina da fêmea e ainda pela interação de ambas, há diversos mecanismos pelos quais a nutrição na fase pré-cobertura poderia afetar a taxa de sobrevivência embrionária. Almeida (2000) observou sobrevivência embrionária reduzida $(\mathrm{P}=0,038)$, mesmo na ausência de efeitos sobre taxa ovulatória, número e tamanho dos embriões, quando as marrãs foram submetidas à restrição alimentar na fase luteal tardia. Kemp et al. (1995) não encontraram efeito da fonte de energia sobre a sobrevivência embrionária, mas relataram aumento nos níveis de progesterona no periestro e aumento na secreção pulsátil do $\mathrm{LH}$, em porcas alimentadas com dieta baseada em amido, em relação ao grupo tratado com dieta essencialmente lipídica. Os dados da Tab. 5 mostram, portanto, que houve efeito das fontes de energia sobre a atividade ovariana. A dieta flushing baseada em amido de milho (T1) aumentou a taxa ovulatória $(\mathrm{P}=0,0052)$. No presente trabalho, entretanto, não houve efeito da leitegada de origem (litter effect) sobre a taxa de ovulação, conforme demonstrado por Almeida (2000).

As respostas podem ser mais amplamente compreendidas quando se avaliam os dados uterinos, placentários e hormonais conjuntamente, os quais representam seqüência ao presente trabalho. Entretanto, há que se registrar que os efeitos obtidos sobre a taxa ovulatória foram alcançados na ausência de diferença em níveis energéticos ou mesmo em padrões de consumo, entre os tratamentos. O modelo experimental utilizado neste experimento procurou, tanto quanto possível, isolar os efeitos da fonte de energia sobre as respostas reprodutivas. $\mathrm{O}$ balanceamento por linhagem genética, idade, origem sanitária, manejo, família (leitegada de origem), peso de entrada, número de cios, espessura de toucinho, profundidade de lombo, saúde clínica, padrões de consumo durante a fase de crescimento, tempo de gestação ao abate, dentre outros critérios utilizados na metodologia deste trabalho, provavelmente contribuiu sobremaneira para a obtenção de coeficientes de variação relativamente baixa neste estudo, se for considerada a variabilidade intrínseca das respostas medidas. Uma vez que não houve variação na ingestão de nutrientes nem tampouco no consumo de alimento diário, entre os tratamentos, as diferenças observadas na resposta reprodutiva devem ser explicadas por modificações metabólicas e/ou hormonais induzidas pela diferença nas fontes de energia aplicadas.

Historicamente, diversos autores já confirmaram os efeitos do flushing nutricional pré-cobertura sobre algumas respostas reprodutivas em leitoas (Flowers et al., 1989; Ashworth et al., 1999; Ferguson et al., 2003). Dentro dos limites alcançados por esta revisão, todavia, não se teve conhecimento de outros modelos experimentais como o proposto neste trabalho, direcionado especificamente para avaliar os efeitos de diferentes fontes de energia sobre a eficiência reprodutiva em marrãs cíclicas e francamente anabólicas. Os trabalhos de Kemp et al., (1995), van den Brand (2000) e van den Brand (2001) dedicaram-se ao estudo dos efeitos das fontes de energia, isoladamente, em porcas primíparas lactantes, portanto catabólicas e sujeitas a outros padrões de regulação hormonal, metabólica e neuroendócrina.

\section{CONCLUSÃO}

A utilização do flushing nutricional nas duas últimas semanas antes da cobertura, como mecanismo de otimização da eficiência reprodutiva de marrãs, pode ser potencializada pela utilização de dietas ricas em carboidratos como fonte predominante de energia. 


\section{REFERÊNCIAS BIBLIOGRÁFICAS}

ALMEIDA, F.R.C.L. Nutrition-reproduction interactions in cyclic gilts. 2000. 263p. PhD Thesis (Doctor of philosophy in Animal Science) - University of Alberta, Edmonton - AB, Canadá.

ALMEIDA, F.R.C.L.; MAO, J.; NOVAK, S. et al. Effects of different patterns of feed restriction and insulin treatment during the luteal phase on reproductive, metabolic, and endocrine parameters in cyclic gilts. J. Anim. Sci., v.79, p.200-212, 2001.

ASHWORTH, C.J.; BEATTIE, L.; ANTIPATIS, C. Effects of pre- and post-mating nutritional status on hepatic function, progesterone concentration, uterine protein secretion and embryo survival in Meishan pigs. Reprod. Fertil. Dev., v.11, p.67-73, 1999.

AZAIN, M.J. Fat in swine nutrition. In: LEWIS, A.J.; SOUTHERN, L.L. (Eds). Swine nutrition. 2.ed. New York: CRC Press, 2001. p.95-105.

BELTRANENA, E.; AHERNE, F.X.; FOXCROFT, G.R. Innate variability in sexual development irrespective of body fatness in gilts. J. Anim. Sci., v.71, p.471-480, 1993.

BRUSTOLINI, P.C.; SILVA, F.C.O.; DONZELE, J.L. et al. Efeitos de diferentes fontes lipídicas e níveis de energia sobre o desempenho reprodutivo de marrãs. Arq. Bras. Med. Vet. Zootec., v.56, p.511-521, 2004.

FERGUSON, E.M.; ASHWORTH, C.J.; EDWARDS S.A. et al. Effect of different nutritional regimens before ovulation on plasma concentrations of metabolic and reproductive hormones and oocyte maturation in gilts. Reproduction, v.126, p.61-71, 2003.

FLOWERS, B.; MARTIN, M.J.; CANTLEY, T.C. et al. Endocrine changes associated with a dietary-induced increase in ovulation rate (flushing) in gilts. J. Anim. Sci., v.67, p.771-778, 1989.
KEMP, B.; SOEDE, N.M.; HELMOND, F.A. et al. Effects of ennergy source in the diet on reproductive hormones and insulin during lactation and subsequent estrus in multiparous sows. J. Anim. Sci., v.73, p.3022-3029, 1995.

MURGAS, L.D.S. Efeito do consumo de energia na fase pré-puberal sobre os desempenhos produtivo e reprodutivo de marrãs. 1994. 65p. Dissertação (Mestrado) - Universidade Federal de Viçosa, Viçosa, MG.

RHODES, M.T.; DAVIS, D. L.; STEVENSON, J.S. Flushing and altrenogest affect litter traits in gilts. J. Anim. Sci., v.69, p.34-40, 1991.

RODRIGUEZ-MARQUEZ, M.C.; CUARON, J.A. Dietary energy source on ovulation in swine. J. Anim. Sci., v.68, suppl. I, p. 367, 1990. (abstract).

SISTEMA de analyses estatísticas. Versão 9.0 SAEG. Viçosa: UFV, 2004. 150p.

van den BRAND, H.; LANGENDIJK, P.; SOEDE, N.M. et al. Effects of postweaning dietary energy source on reproductive traits in primiparous sows. J. Anim. Sci., v.79, p.420-426, 2001.

van den BRAND, H. Energy partitioning and reproduction in primiparous sows: effects of dietary energy source. 2000. 139p. PhD Thesis. Wageningen Institute of Animal Sciences, Wageningen University.

WELDON, W.C.; LEWIS, A.J.; LOUIS, G.F. et al. Postpartum hypophagia in primiparous sows: II. Effects of feeding level during gestation and exogenous insulin on lactation feed intake, glucose tolerance, and epinephrine-stimulated release of nonesterified fatty acids and glucose. J. Anim, Sci., v.72, p.395-403, 1994.

WHITTEMORE, C. The science and practice of pig production. Oxford: Blackwell Science, 1998. 624p. 